\title{
Precise alcoholic Gasserian injection for tic douloureux ${ }^{1}$
}

\author{
ARTHUR ECKER AND THEODORE PERL
}

\author{
From the Departments of Surgery (Neurosurgery) and of Radiology, State University of New York, Upstate \\ Medical Center, Syracuse, New York
}

Since October 1954 we have been treating major paroxysmal trigeminal neuralgia (tic douloureux) by precise alcoholic injection of the trigeminal root and ganglion (Ecker and Perl, 1958). To reach the ganglion the needle enters the anterior part of the cheek and proceeds through the foramen ovale. With Penman's (1953) radiographic technique we determine the exact relation of the intracranial tip of the needle to the foramen ovale. We then inject the presumed junction of posterior root and ganglion with minute amounts, each $0.05 \mathrm{ml}$, of absolute alcohol. Our goal is to produce permanent anaesthesia to light touch in the trigger area of lips and jaws, while sparing corneal sensation as advised by Kugelberg and Lindblom (1959).

In most cases, relief of pain has been immediate and has lasted for years (Fig. 1). In cases where, later, anaesthesia disappeared and pain recurred, a second injection was soon requested and promptly brought prolonged relief.

We recognize dangers such as corneal anaesthesia with its risk of keratitis, meningitis, and cranial nerve palsies. A few of our early patients have had such complications but the residua are rare and mild.

Over an eight-year period, we have managed 133 cases with a total of 173 sessions of injection. We have kept our patients free from severe pain and have produced no permanent serious sequelae and no deaths. Nevertheless, in this paper we emphasize the complications.

We began this work in October 1954. Eight years later, in November-December 1962, we carried out a follow-up study of 68 of the 73 patients injected before 23 October 1959. Thus, this is a detailed study of those 68 cases, three to eight years after injection. We also present a summary of the present status of $111(93 \%)$ of 119 patients, a year or more after injection.

\section{CASE MATERIAL}

This report is based on 133 consecutive cases of major

${ }^{1}$ Presented at the Mayo Clinic, Rochester, Minnesota on the 50th anniversary of the founding of the section of Neurology, 9 February 1963. paroxysmal trigeminal neuralgia (tic douloureux) treated by precise alcoholic injection at the juncture of the Gasserian ganglion and its posterior root. Fourteen of our patients had had previous intracranial rhizotomy, but the pain had recurred. Most of the patients referred to us had already tried diphenylhydantoinate sodium or mephenesin carbamate but pain persisted or recurred. A few others, those with mild pain, were advised by us to try these drugs. When pain was severe or medical therapy failed, we recommended alcoholic Gasserian injection. All but two patients accepted our recommendation. Essentially, this is a report on a consecutive series of cases with severe tic douloureux. Most patients were elderly, and many were poor surgical risks. However, no one was excluded because of risk or any other associated medical condition.

\section{METHOD}

Our technique has evolved over eight years and the details have been published elsewhere $(1958,1959,1963)$. Here is a summary.

The procedure is performed on out-patients who have omitted the previous meal. Preliminary radiography determines the position for the patient's head and the entry point of the needle. Then a needle, directed vertically downward, enters the foramen ovale. To provide some local anaesthesia for the course of the needle $20 \mathrm{ml}$. of lidocaine (Xylocaine) hydrochloride $1 \%$ is injected.

This fluid also makes the cheek turgid so that the skin is less likely to move while it is marked and while the final needle is inserted. Thirdly, the injected fluid depresses the oral mucous membrane near the coronoid process of the mandible, and permits the needle to remain lateral to the oral cavity (Fig. 1).

The radiographic table is horizontal. The patient lies supine on a mattress with his head extended beyond it. With biplane guidance, a needle directed downward is inserted just through the foramen ovale (Fig. 2). The depth of the needle is controlled either by the appearance of cerebrospinal fluid, which signals the junction of ganglion and posterior root, or by placement of the needle at the apparent depth of 4 to $5 \mathrm{~mm}$. according to submentovertical radiographs. This apparent depth of the needle beyond the foramen ovale is judged by the radiolucent distal portion of the needle after the stylet has been withdrawn exactly $10 \mathrm{~mm}$. from the hub (Penman, 1953). When the depth of the needle is corrected, the face is 


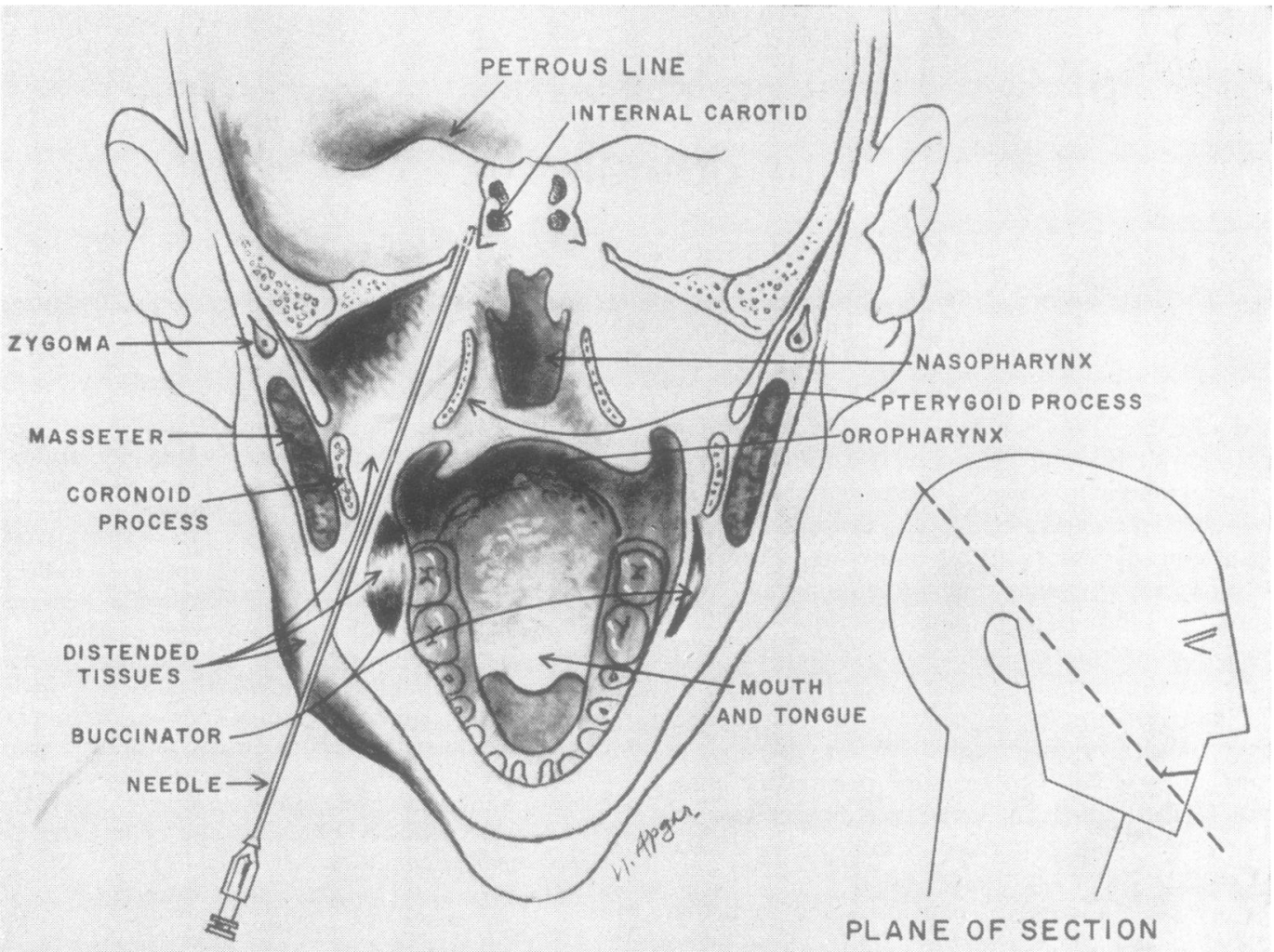

FIG. 1

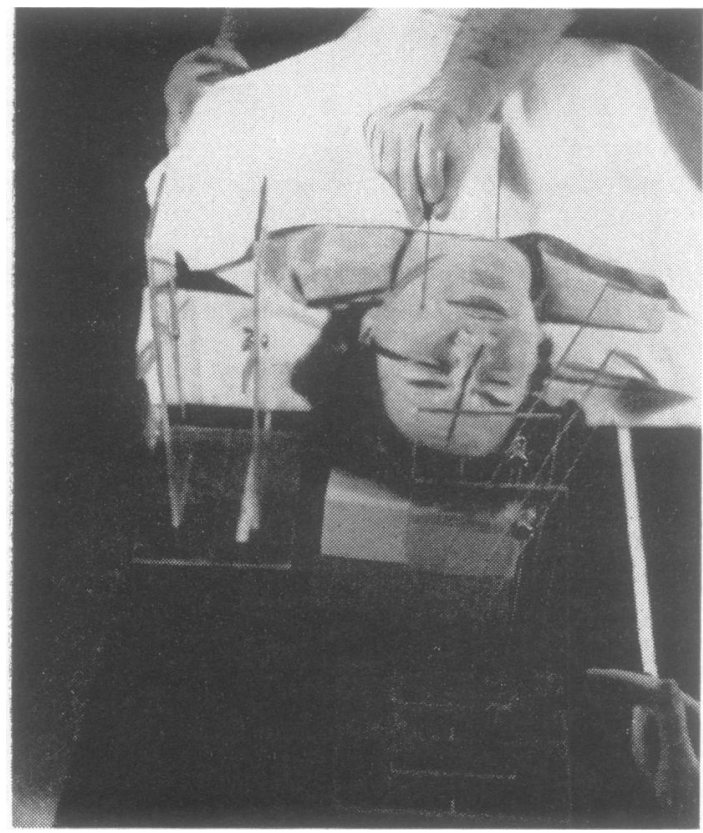

FIG. 1. Effect of preliminary lidocaine injection. Final $\stackrel{\overrightarrow{7}}{0}$ needle in place.

FIG. 2. Cheek and final entry point marked on skin. Biplane guidance of final needle.

FIG. 2 
rotated further toward the healthy side and the hyperextension of the head is relieved. This keeps the corneal fibres low in both root and ophthalmic division. Above these corneal fibres, the alcohol rises in Meckel's cavity, the meningeal container of the Gasserian ganglion. Exactly $0.05 \mathrm{ml}$. of absolute alcohol is injected. Careful sensory tests are made and recorded. Fortunately, maximal sensory defect is often produced in the trigger areas; after adequate injection, even firm pressure on these areas is painless. The anaesthesia of the maxillary and/or mandibular division should persist for 40 minutes and the injection is repeated until it does. Then, both the sensory defect and relief of pain are likely to be permanent.

After the procedure the patient is permitted to go home. If cerebrospinal fluid has escaped, he is advised to keep the head low for 12 hours. If corneal anaesthesia has been produced, the patient is cautioned not to rub his eye. He reports for re-examination in one or two days.

\section{RESULTS AFTER THREE TO EIGHT YEARS}

At follow-up after three years most of the patients were still free of pain and grateful. The accompanying graphs (Fig. 3) show the relief of trigeminal neuralgia. They are designed to indicate pain over a period of years, both before and after alcoholic Gasserian injection. Pain before the injection was reported by the patients, or by a relative, at the time of the injection.

Of the 73 patients injected more than three years ago, case 7 is excluded because the needle had not been inserted through the foramen ovale. Case 8 was relieved of pain for only a few days, and received rhizotomy elsewhere. Case 31 is the only one lost to follow-up. Case 34 is a 71-year-old patient who had immediate and persisting relief of pain, and who died of coronary occlusion three weeks later. Case 65 was a 55-year-old worman relieved of trigeminal neuralgia for a month, who died suddenly of a parieto-occipital cerebral haemorrhage; at necropsy it was shown to be unrelated to the base of the skull and brain. Case 69 is excluded because it was not trigeminal neuralgia, but carcinoma of the antrum (where relief was obtained for the last 10 months of life). In summary, excluded from this detailed followup report are cases, 7, 8, 31, 34, 65, and 69. Patient 55 is listed as two cases (55A and 55B) because there was independent trigeminal neuralgia on each side of his face.

Precise alcoholic Gasserian injection is usually effective in relieving tic douloureux. Figure 3 shows that with the present programme, patients have been largely free of trigeminal pain for several years. From 68 cases followed for at least three years, the following data have been derived: at follow-up, 25 cases had no pain and no paraesthesia; most of these patients had subjective awareness of anaesthesia which they usually called numbness. Of the 68 patients, $49(72 \%)$ remained free of severe pain for over three years following a single treatment. Nineteen required additional injections. Of these, eight had a second injection within two months and 11 after two months. Five of the 68 required three injections. The charts demonstrate that if severe pain recurred the patients promptly requested another Gasserian injection.

At follow-up no patient was having severe trigeminal pain. However, six (cases 3, 24, 29, 39, 63, and 70) complained of intermittent mild pain of the trigeminal type, sometimes partly controlled by diphenylhydantoinate sodium.

\section{COMPLICATIONS}

DEATHS No deaths resulted from the procedure. However, three patients died of unrelated conditions within a month: cases 34 and 65 are described above, and case 47 is below.

MENINGITIS Three patients developed meningitis, one with demonstrable bacteria and two without. Two of these occurred before we developed our present method of keeping the needle lateral to the oral mucous membrane. All recovered from the attack of meningitis without residual defect.

In case 47 , the patient developed bacterial meningitis eight days after the injection. A cerebrospinal fluid leak had persisted because a glioma of the brainstem had produced increased intracranial pressure. The patient was relieved of pain and had recovered fully from the meningitis. She died of a complication in the later treatment of obstructive hydrocephalus which resulted from the neoplasm.

CORNEAL ANAESTHESIA Eight of these 68 patients suffered corneal anaesthesia and five of the eight developed superficial keratitis. One required tarsorrhaphy, but none of this group lost visual acuity.

PARAESTHESIAE Twenty-seven of the patients now have some paraesthesia. Generally they described it as a crawling, fluttering, or burning sensation. In eight this was severe. The degree of anaesthesia produced was not related to complaints. In recent years, we have inquired about paraesthesia before the injection, and have often found it present. Indeed, one man experienced a tingling in the face which he found pleasant (!) for six months before it became associated with pain. One of the patients who complained most bitterly after the procedure had told of the same severe paraesthesia before it. Nine patients had various pains in the face other than trigeminal neuralgia or paraesthesia. Often these 'other pains' occurred only at times of personal stress. 


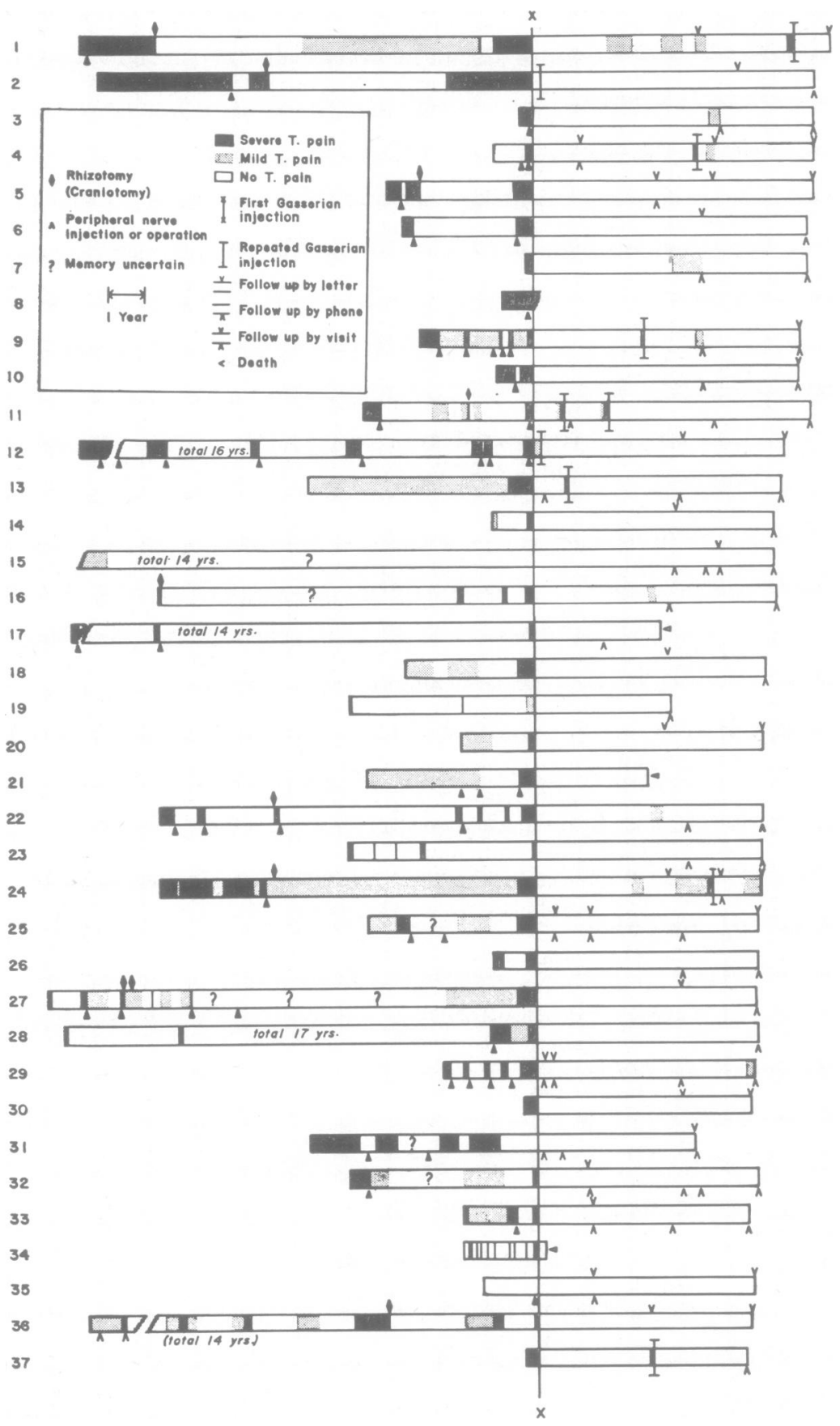

FIG. 3a. Graphic demonstration of trigeminal pain before and after alcoholic Gasserian injection in cases 1-37. 


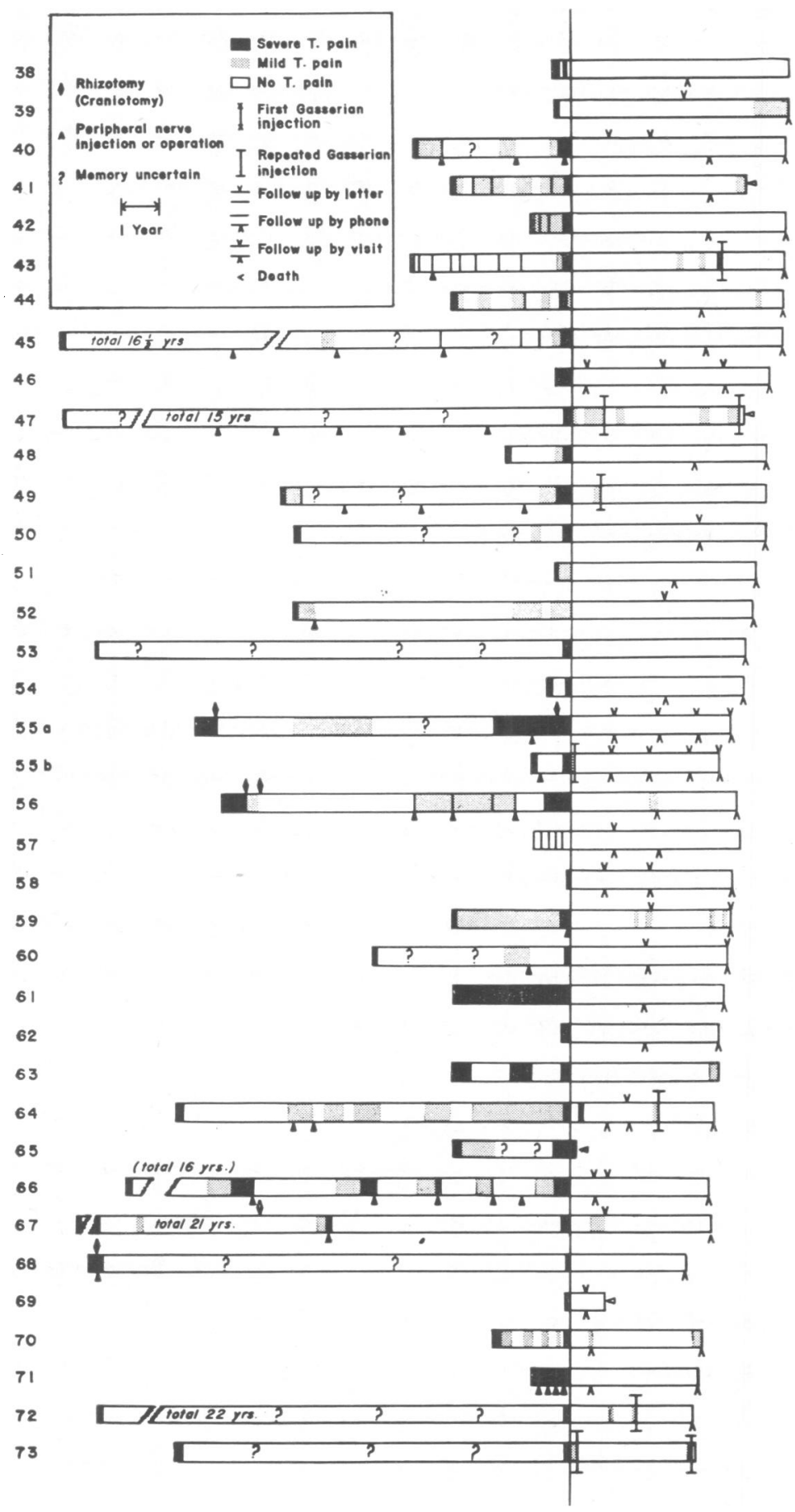

FIG. 3b. Graphic demonstration of trigeminal pain before and after alcoholic Gasserian injection in cases 38-73. 
OTHER COMPLICATIONS Two patients (cases 10 and 58) developed small superficial ulcers of anaesthetic areas of the nostril. These healed promptly when the patients stopped picking the skin. There was one case of minor posterior inferior cerebellar artery syndrome, induced by extending the head for the injection. Presumably it was due to occlusion of the vertebral artery in its cervical course.

Minor complications included headache on the night of injection, usually relieved by aspirin, painless herpes simplex on the lip, and haematoma in the cheek, followed by ecchymosis. Paralysis of the ipsilateral masticator muscles was common but never lasted more than three months.

In our early experience (Ecker and Perl, 1958) there were isolated examples of third, sixth, seventh, and eighth cranial nerve palsies during the procedure. Each lasted only a few minutes. There has been none since limiting the volume of each injection to $0.05 \mathrm{ml}$.

Following Gasserian injection there is frequently loss of sensation to touch and pain on the ipsilateral half of the anterior two-thirds of the tongue. While this sensory defect persists, taste sensation is usually absent in the same area. Many normal people are unable to taste salt or sugar (Stookey and Ransohoff, 1959).

RESULTS AFTER ONE TO EIGHT YEARS More recently we were able to follow $111(93 \%)$ of those 119 patients injected a year or more previously. At this time, only three had trigeminal pain and additional injections may help two of them. Ten $(9 \%)$ had severely distressing paraesthesias.

Thirteen of the entire 133 in the whole series had total corneal anaesthesia; of these, six had had superficial keratitis; two had required temporary tarsorrhaphy and one had diminution of visual acuity to $20 / 100+$ from a corneal scar.

\section{SUMMARY}

In the course of eight years, we have managed 133 successive cases of paroxysmal trigeminal neuralgia (tic douloureux) by precise alcoholic injection into the region of the junction of the Gasserian ganglion and its posterior root. Thirty-six $(27 \%)$ of these patients were 70 years of age or more. Fourteen had had previous craniotomy with rhizotomy.

Our goal is to denervate the trigger areas in the jaws and lips while sparing corneal sensation. Our method is an out-patient procedure. It requires cooperation of radiologist, surgeon, and patient for one to two hours. Its precision is based on radiographic control of insertion of the needle and clinical control of the extent and intensity of the sensory loss produced.

We include a detailed graphic report of relief of pain in the first 68 cases followed for three to eight years (Fig. 3). All patients were relieved of severe trigeminal pain. There were no deaths or other serious permanent complications from the procedure. After a single session $49(72 \%)$ remained free from pain for over three years. Eight $(12 \%)$ required a second injection within two months because inadequate sensory loss was produced by the first. Eleven others $(16 \%)$ required an additional injection after two months. The charts show that in the cases where pain recurred, patients did not suffer long before requesting another injection. Eight patients of the 68 experienced corneal anaesthesia, but none lost vision. Paraesthesias still remain a problem in eight of the cases; some of these cases can be recognized before the injection is made.

A recent follow-up of 111 (93\%) of the 119 patientso. injected a year or more previously revealed three $\mathrm{G}$ with recurrent trigeminal pain and one with visuas 9 acuity reduced to $20 / 100+$ from a corneal scar $\underset{\Omega}{\Phi} \overrightarrow{7}$ 10 of these patients $(9 \%)$ had distressing paraesthesia용 $\mathbb{D}$

This method of management of trigeminal neural gia is effective and safe, even for patients who are poor surgical risks.

We are grateful to Dr. Albert J. Schneider for advice on the preparation of the graphs, and to Miss Joyce Berman for their production.

\section{REFERENCES}

Ecker, A., and Perl, T. (1958). Alcoholic Gasserian injection for relief of tic douloureux. Preliminary report of a modification of Penman's method. Neurology (Minneap.), 8, 461-468.

Kugelberg, E., and Lindblom, U. (1959). The mechanism of the pain in trigeminal neuralgia. J. Neurol. Neurosurg. Psychiat., 22, $36-43$.

Penman, J. (1953). Some developments in the technique of trigemina injection. Lancet, $1,760-764$.

Perl, T., and Ecker, A. (1959). Roentgenologically controlled place ment of the needle in the trigeminal root for the treatment of tic douloureux. Amer. J. Roentgenol., 82, 830-839. , (1963). Radiologically controlled injections through the foramen ovale for relief of tic douloureux and of Parkinsonism. Acta radiol. (Stockh.), n.s. 1, 901-912.

Stookey, B., and Ransohoff, J. (1959). Trigeminal Neuralgia: it History and Treatment, p. 83. Thomas, Springfield, Illinois. 